\title{
Inventaires préliminaires des oiseaux de la forêt des marais Tanoé-Ehy en Côte d'Ivoire
}

\author{
Dibié Bernard $\mathrm{AHON}^{1 *}$, Wadja Mathieu EGNANKOU ${ }^{2}$, Koffi Roger KOUADIO ${ }^{3}$ et \\ Odé Marie Louise KOUAME ${ }^{2}$ \\ ${ }^{1}$ Unité Pédagogique et de Recherche de Biologie et Physiologie Animale, URES Daloa, \\ BP 150 Daloa, Côte d'Ivoire. \\ ${ }^{2}$ Laboratoire de Botanique, UFR Biosciences, Université de Cocody Abidjan, 22 BP 582 Abidjan 22, \\ Côte d'Ivoire. \\ ${ }^{3}$ Laboratoire de Zoologie et Biologie Animale, UFR Biosciences, Université de Cocody, Abidjan, \\ 22 BP 582 Abidjan 22, Côte d'Ivoire. \\ *Auteur correspondant, E-mail: bahon2002@yahoo.fr; bdibie2@yahoo.fr; \\ Tel. : + $22507523439 /+22503373914$
}

\section{RESUME}

L'avifaune de la forêt des marais Tanoé-Ehy (FMTE) était jusqu'alors méconnue des ornithologues. Des inventaires préliminaires des oiseaux de ce site ont été réalisés en mai/juin 2008 (saison pluvieuse) et mars 2010 (saison sèche) afin de dresser la liste des espèces d'oiseaux existantes, d'identifier les espèces rares ou en voie de disparition et de déterminer les flux migratoires des principales espèces. Différentes méthodes dont l'observation des oiseaux par une marche lente le long des pistes et sentiers existants dans la forêt, la progression lente à bord d'une pirogue, l'observation des oiseaux à partir d'un point fixe (à haute altitude) de la forêt, les techniques de repasse de vocalisation à partir des stations d'écoute et de captures au filet japonais, ont été mises en œuvre dans différents habitats. Au total, 274 espèces d'oiseaux de 58 familles ont été recensées. Douze de ces espèces sont incluses dans la liste des espèces dont la protection est d'intérêt mondial et sept d'entre elles ont été identifiées comme espèces à répartition restreinte. La découverte de la Chouette-pêcheuse de Bouvier Scotopelia bouvieri (nouvelle espèce pour la Côte d'Ivoire) est l'un des résultats les plus intéressants de cette étude. Il est probable que la liste des espèces s'allonge lorsque l'étude de la forêt sera complète.

() 2012 International Formulae Group. All rights reserved

Mots clés: Inventaires, oiseaux, Forêt marais Tanoé-Ehy, Côte d'Ivoire.

\section{INTRODUCTION}

L'écosystème forestier de la Haute Guinée de l'Afrique de l'Ouest représente l'un des points les plus importants de la biodiversité au monde (Meyers et al., 2000) et une haute priorité pour sa conservation. Les oiseaux occupent une place importante au sein de cette biodiversité forestière (Bennun et al., 2004). Ils contribuent activement au fonctionnement des écosystèmes (pollinisation, dissémination des graines, etc.). De par leur visibilité, l'étendue et la diversité de leurs habitats, de même que de par leur rôle écologique, ils sont d'importants indicateurs 
de la santé des écosystèmes (Demey et Rainey, 2005). Ils sont ainsi utilisés pour le suivi des changements écologiques (Furness et Greenwood, 1993) ou pour l'évaluation de l'importance de la diversité biologique (Thirgood et Heath, 1994 ; Stattersfield et al., 1998). Leur taxinomie et leur répartition géographique mondiale sont relativement bien documentées en comparaison à d'autres taxons (Icbp, 1992), ce qui facilite leur identification et permet l'analyse rapide des résultats d'une étude ornithologique. Le statut de conservation de la plupart des espèces ayant été assez bien évalué, les résultats et conclusions d'une telle étude peuvent être évalués et mis à exécution de façon positive.

Située dans une zone de grande importance pour la conservation de la biodiversité, la Forêt des Marais Tanoé-Ehy (FMTE) a été identifiée comme étant d'une importance exceptionnelle parmi les aires prioritaires de conservation régionale pour la forêt de Haute Guinée et d'une haute importance pour la conservation des mammifères et des oiseaux (Conservation International, 2001). Ce site représente le dernier refuge viable pour bon nombre de représentants de la faune originelle du sud-est de la Côte-d'Ivoire (Béné et al., 2012).

Malgré cette importance capitale, la FMTE n'a fait l'objet d'aucune étude ornithologique avant la présente étude. Pour pallier à ce manque de connaissances, nous avons effectué des inventaires de la faune aviaire dudit site en 16 jours de travail sur le terrain dont 5 jours en saison pluvieuse (du 27 mai 2008 au 01 juin 2008) et 11 jours en saison sèche (du 18 au 28 mars 2010).

Les présents inventaires préliminaires se proposent donc d'approfondir les connaissances sur la faune aviaire de la FMTE en vue d'évaluer un peu plus, la valeur de conservation de cette forêt. Les principaux objectifs de cette étude sont; (i) évaluer la diversité et l'abondance de la faune aviaire de la FMTE; (ii) dresser la liste des espèces d'oiseaux existantes en identifiant les espèces rares ou en voie de disparition ; (iii) identifier les espèces endémiques, à répartition restreinte et/ou appartenant à un biome particulier et enfin; (iv) déterminer les flux migratoires et indiquer si la FMTE peut être qualifiée comme Zone Importante pour la Conservation des Oiseaux (ZICO) selon les critères définis par Fishpool et Evans (2001).

\section{MATERIEL ET METHODES \\ Description du site}

La FMTE est située au sud-est de la Côte-d'Ivoire entre la lagune Ehy (à l'ouest) et le fleuve Tanoé (au sud et à l'est) (Figure 1). Elle est située dans le Département de Tiapoum et dans les Sous-préfectures de Noé et Nouamou. Seul bloc de forêt encore relativement intact dans la région du sud-est de la Côte d'Ivoire, la FMTE est localisée entre $5^{\circ} 05^{\prime}$ et $5^{\circ} 15^{\prime}$ de latitude nord et $2^{\circ}$ $45^{\prime}$ et $5^{\circ} 53^{\prime}$ de longitude ouest (Adou, 2007). Les précipitations moyennes annuelles sont de $1500 \mathrm{~mm}$ et les températures oscillent annuellement entre $22{ }^{\circ} \mathrm{C}$ et $30{ }^{\circ} \mathrm{C}$ (Adou, 2007).

La FMTE est recouverte d'une végétation de forêt sempervirente marécageuse sur sol hydromorphe (Adou, 2007) au niveau du littoral de la Côte d'Ivoire. Le groupement caractéristique de cette forêt est le groupement à Mitragyna ciliata et Symphonia globulifera. A l'image des « Hotspots » de la Haute Guinée, la flore de la FMTE est très diversifiée avec une richesse remarquable. Sa faune simienne est également riche et exceptionnelle. On y rencontre des singes rares et menacés d'extinction tels que Piliocolobus badius waldronae, Colobus vellerosus, Cercopithecus diana roloway, Procolobus verus, Cercopithecus campbelli lowei, Cercopithecus petaurista petaurista, Cercocebus atys lunulatus et Pan troglodytes (Béné et al., 2012).

\section{Recueil des données sur les oiseaux}

La principale méthode utilisée pendant cette étude a consisté en l'observation des oiseaux en marchant lentement $(0,5 \mathrm{~km} / \mathrm{h}$ au maximum) le long des pistes et des sentiers existants dans les différents habitats. Des notes ont été prises sur les observations 

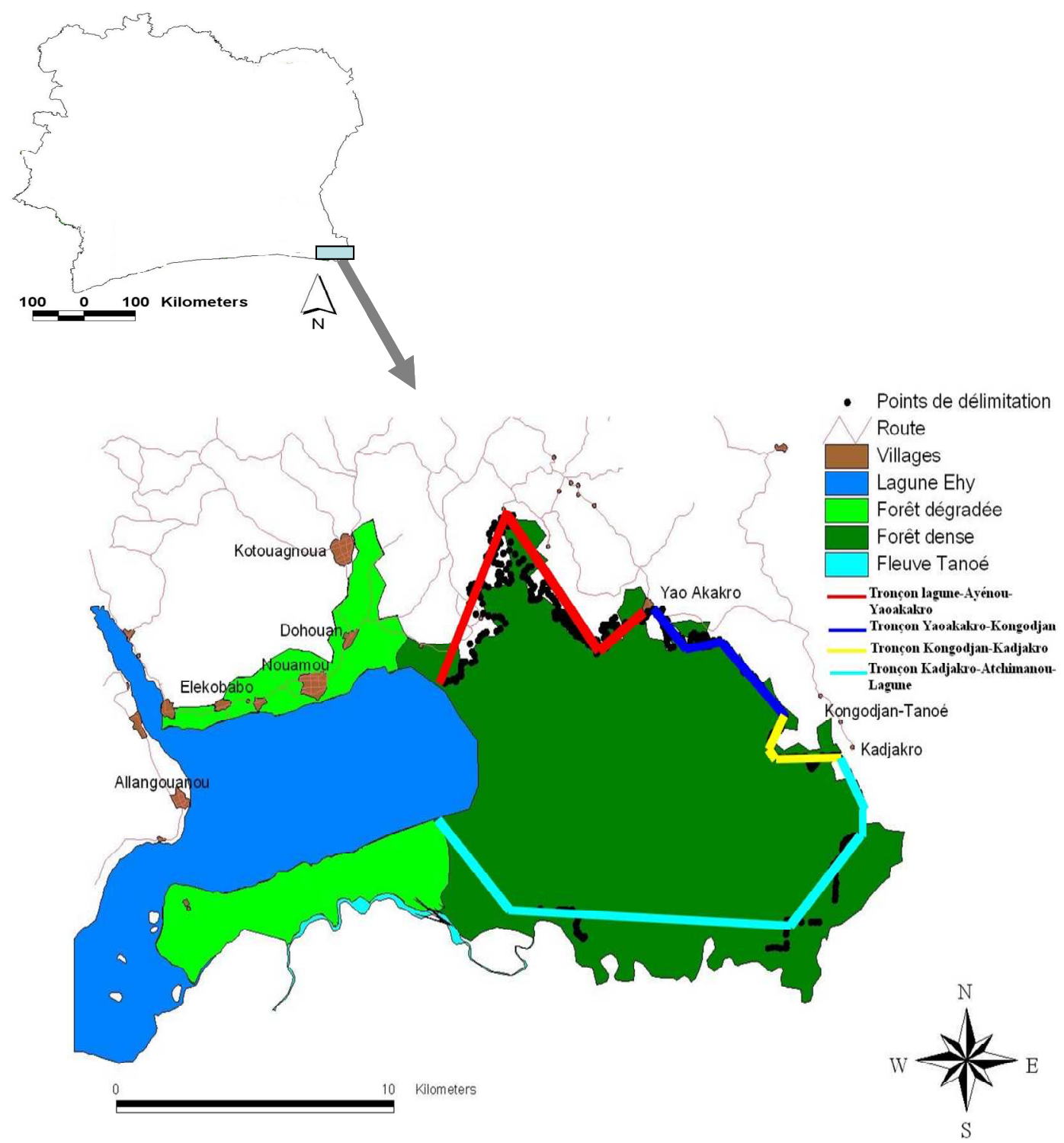

Figure 1: Localisation de la forêt des marais Tanoé-Ehy au sud-est de la Côte d'Ivoire.

visuelles et les émissions vocales des oiseaux. L'enregistreur de son (le dictaphone) a été utilisé, soit pour enregistrer les chants et cris des oiseaux inconnus qui ont été identifiés plus tard au Laboratoire grâce aux CD-ROM de Claude CHAPPUIS (Chappuis, 2000), soit jouer en play-back (repasse de vocalisation) afin de permettre aux espèces éloignées de s'approcher de nous pour leur meilleure observation et identification. Le travail sur le terrain était effectué généralement de 06 h 30 à $11 \mathrm{~h} 00$ et de $15 \mathrm{~h}$ à $18 \mathrm{H} 00$. Six sorties nocturnes ont été effectuées (en zones de forêt) afin de collecter des données sur les hiboux et les engoulevents, et d'enregistrer leurs vocalisations. Sur ces six sorties nocturnes, trois ont été effectuées de 19 h 00 à $23 \mathrm{~h} 00$ et trois autres, de $04 \mathrm{~h}$ à 06 h 00 du matin. Des captures aux filets japonais ont été faites pendant la journée (de $07 \mathrm{~h}$ à $18 \mathrm{~h}$ ) et la 
nuit (de $19 \mathrm{~h}$ à $23 \mathrm{~h}$ ) à trois reprises dans trois différents secteurs de la FMTE, soit une durée totale de capture de 45 heures. L'un des deux filets était à chaque fois placé en zone de forêt en bon état de conservation et l'autre, dans une parcelle de jachère à la limite forêt/jachère. Ces deux filets japonais avaient une longueur de $12 \mathrm{~m}$ chacun (soit $24 \mathrm{~m}$ linéaires de filets placés dans chaque secteur). L'objectif principal de ces captures était d'obtenir des données sur des espèces discrètes et silencieuses qui peuvent facilement passer inaperçues lors des observations visuelles ou auditives. Une demijournée a été consacrée à une observation le long de la rivière «Douhognan » par une progression lente à bord d'une pirogue à partir $\mathrm{du}$ point de coordonnées géo-référentiels $0520126 \mathrm{~N} / 0573475 \mathrm{O}$ (point de départ) jusqu'à celui de 0523170 N / $057554 \mathrm{O}$ (point d'arrivée). Des observations à partir d'un point fixe à haute altitude « la plate-forme (0519215 N / 0574988 O) » ont été faites afin d'observer les éventuelles espèces d'oiseaux notamment les rapaces qui pourraient utiliser ce beau site comme voie migratoire.

Chaque jour, une liste exhaustive des espèces observées a été établie. Le nombre d'individus ou de groupes a été noté. Ceci nous a permis de produire l'indice d'abondance basé sur le taux de rencontre (nombre de jours pendant lesquels l'espèce fut notée et nombre d'individus et de groupes concernés). La nomenclature suit celle de Borrow et Demey (2001) et les catégories d'abondance utilisées sont celles de Demey et Rainey (2005).

\section{RESULTATS}

Toutes méthodes confondues et sur l'ensemble des deux visites (saison pluvieuse et saison sèche), 274 espèces d'oiseaux (dont une nouvelle mention pour la Côte d'Ivoire: la Chouette-pêcheuse de Bouvier Scotopelia bouvieri) de 58 familles ont été recensées (Tableau 1). La Figure 2 présente les photos de quelques espèces d'oiseaux observées dans la FMTE.

Le peuplement d'oiseaux de la zone d'étude est caractérisé par $38 \%$ d'espèces communes, $33 \%$ d'espèces assez communes, $23 \%$ d'espèces peu communes et $6 \%$ d'espèces rares. Douze espèces recensées, bénéficient d'une protection d'intérêt mondial (BirdLife International, 2010). Il s'agit d'une espèce en danger (EN) : la Chouette-pêcheuse rousse Scotopelia ussheri; de quatre espèces vulnérables (VU) : le Circaète de Baudouin Circaetus baudouini, l'Echenilleur à barbillons Lobotus lobatus, le Bulbul à tache jaune Criniger olivaceus, le Bulbul à queue verte Bleda eximius; de six espèces quasimenacées (NT): le Busard pâle Circus macrourus, le Perroquet jacko Psittacus erithacus, le Calao à casque jaune Ceratogymna elata, le Bathmocerque à capuchon Bathmocercus cerviniventris, l'Akalat à ailes rousses Illadopsis rufescens, le Choucador à queue bronzée Lamprotornis cupreocauda et d'une espèce insuffisamment documentée (DD) : l'Indicateur d'Eisentraut Melignomon Eisentrauti.

A partir des statuts biogéographiques des différentes espèces définies dans la liste des espèces, il ressort que $17,51 \%$ des espèces recensées sont migratrices dont $9,12 \%$ d'espèces migratrices Paléarctiques et 8,39\% d'espèces migratrices Intra- africain.

Parmi les espèces recensées, huit sont à répartition restreinte, c'est-à-dire, des espèces d'oiseaux terrestres dont l'aire de reproduction est inférieure à $50000 \mathrm{~km}^{2}$ qui composent la Zone d'Endémisme d'Oiseaux des forêts de Haute Guinée. Quinze espèces inventoriées sont endémiques à l'Afrique de l'Ouest.

Quant aux biomes, il a été observé 110 espèces d'oiseaux du biome des forêts Guinéo-Congolaises et quatre espèces de la savane soudano-guinéenne connues en Côte d'Ivoire. 
Tableau 1: Liste des espèces d'oiseaux identifiées dans la forêt des marais Tanoé-Ehy.

\begin{tabular}{|c|c|c|c|c|c|c|}
\hline Nom scientifique & Nom en Français & Obs. & Ab. & Cons. & Migr. Endém. & Biome \\
\hline \multicolumn{7}{|l|}{ Phalacrocoracidae } \\
\hline Phalacrocorax africanus & Cormoran africain & $\mathrm{V}$ & $\mathrm{F}$ & $\mathrm{LC}$ & $\mathrm{R}$ & \\
\hline \multicolumn{7}{|l|}{ Anhingidae } \\
\hline Anhinga rufa & Anhinga d'Afrique & $\mathrm{V}$ & $\mathrm{F}$ & $\mathrm{LC}$ & $\mathrm{R}$ & \\
\hline \multicolumn{7}{|l|}{ Ardeidae } \\
\hline Ixobrychus minutus & Blongios nain & V & $\mathrm{F}$ & $\mathrm{LC}$ & $\mathrm{R}$ & \\
\hline Ixobrychus sturmii & Blongios de Sturm & $\mathrm{V}$ & $\mathrm{F}$ & $\mathrm{LC}$ & $\mathrm{P}$ & \\
\hline Tigriornis leucolopha & Onoré à huppe blanche & VP & $\mathrm{F}$ & $\mathrm{LC}$ & $\mathrm{R}$ & GC \\
\hline Nycticorax nycticorax & Bihoreau gris & $\mathrm{V}$ & $\mathrm{F}$ & $\mathrm{LC}$ & $\mathrm{R}$ & \\
\hline Ardeola ralloides & Crabier chevelu & $\mathrm{V}$ & $\mathrm{F}$ & $\mathrm{LC}$ & $\mathrm{R} / \mathrm{P}$ & \\
\hline Bubulcus ibis & Héron garde-bœufs & VP & $\mathrm{F}$ & $\mathrm{LC}$ & $\mathrm{R} / \mathrm{M}$ & \\
\hline Butorides striata & Héron strié & VP & $\mathrm{F}$ & $\mathrm{LC}$ & $\mathrm{R}$ & \\
\hline Egretta ardesiaca & Aigrette ardoisée & & $\mathrm{F}$ & $\mathrm{LC}$ & $\mathrm{R}$ & \\
\hline Egretta gularis & Aigrette à gorge blanche & $\mathrm{V}$ & $\mathrm{U}$ & $\mathrm{LC}$ & $\mathrm{R}$ & \\
\hline Egretta garzetta & Aigrette garzette & $\mathrm{V}$ & $\mathrm{U}$ & $\mathrm{LC}$ & $\mathrm{R}$ & \\
\hline Ardea purpurea & Héron pourpré & V & $\mathrm{U}$ & $\mathrm{LC}$ & $\mathrm{R} / \mathrm{P}$ & \\
\hline Ardea cinerea & Héron cendré & $\mathrm{V}$ & $\mathrm{U}$ & $\mathrm{LC}$ & $\mathrm{R} / \mathrm{P}$ & \\
\hline \multicolumn{7}{|l|}{ Ciconiidae } \\
\hline Ciconia abdimii & Cigogne d'Abdim & $\mathrm{V}$ & $\mathrm{F}$ & $\mathrm{LC}$ & M & \\
\hline Ciconia episcopus & Cigogne épiscopale & $\mathrm{V}$ & $\mathrm{F}$ & $\mathrm{LC}$ & $\mathrm{R}$ & \\
\hline \multicolumn{7}{|l|}{ Threskiornithidae } \\
\hline Bostrychia hagedash & Ibis hagedash & $\mathrm{V}$ & $\mathrm{U}$ & $\mathrm{LC}$ & $\mathrm{R}$ & \\
\hline \multicolumn{7}{|l|}{ Anatidae } \\
\hline Dendrocygna viduata & Dendrocygne veuf & VP & $\mathrm{F}$ & $\mathrm{LC}$ & $\mathrm{R}$ & \\
\hline Pteronetta hartlaubii & Canard de Hartlaub & V & $\mathrm{C}$ & $\mathrm{LC}$ & $\mathrm{R}$ & GC \\
\hline Nettapus auritus & Anserelle naine & V & $\mathrm{F}$ & $\mathrm{LC}$ & $\mathrm{R}$ & \\
\hline \multicolumn{7}{|l|}{ Pandionidae } \\
\hline Pandion haliaetus & Balbuzard pêcheur & VA & $\mathrm{U}$ & $\mathrm{LC}$ & $\mathrm{P}$ & \\
\hline \multicolumn{7}{|l|}{ Accipitridae } \\
\hline Aviceda cuculoides & Baza coucou & $\mathrm{V}$ & $\mathrm{U}$ & $\mathrm{LC}$ & $\mathrm{R}$ & \\
\hline Pernis apivorus & Bondrée apivore & $\mathrm{V}$ & $\mathrm{U}$ & $\mathrm{LC}$ & $\mathrm{P}$ & \\
\hline Macheiramphus alcinus & Milan des chauves-souris & $\mathrm{V}$ & $\mathrm{F}$ & $\mathrm{LC}$ & $\mathrm{R}$ & \\
\hline Elanus caeruleus & Elanion blanc & $\mathrm{V}$ & $\mathrm{U}$ & $\mathrm{LC}$ & $\mathrm{R}$ & \\
\hline Milvus migrans & Milan noir & VAP & $\mathrm{C}$ & $\mathrm{LC}$ & M & \\
\hline Haliaeetus vocifer & Pygargue vocifer & $\mathrm{V}$ & $\mathrm{Ra}$ & $\mathrm{LC}$ & $\mathrm{R}$ & \\
\hline Gypohierax angolensis & Palmiste africain & VA & $\mathrm{F}$ & $\mathrm{LC}$ & $\mathrm{R}$ & \\
\hline Circaetus beaudouini & Circaète de Beaudouin & $\mathrm{V}$ & $\mathrm{Ra}$ & VU & $\mathrm{R}$ & \\
\hline Dryotriorchis spectabilis & Serpentaire du Congo & $\mathrm{V}$ & $\mathrm{U}$ & $\mathrm{LC}$ & $\mathrm{R}$ & GC \\
\hline Polyboroides typus & Gymnogène d'Afrique & VAP & $\mathrm{F}$ & $\mathrm{LC}$ & $\mathrm{R}$ & \\
\hline Circus macrourus & Busard pâle & $\mathrm{V}$ & $\mathrm{Ra}$ & NT & $\mathrm{P}$ & \\
\hline Circus aeruginosus & Busard des roseaux & $\mathrm{V}$ & $\mathrm{U}$ & $\mathrm{LC}$ & $\mathrm{P}$ & \\
\hline Accipiter tachiro & Autour tachiro & $\mathrm{V}$ & $\mathrm{F}$ & $\mathrm{LC}$ & $\mathrm{R}$ & \\
\hline Accipiter badius & Epervier shikra & VA & $\mathrm{F}$ & $\mathrm{LC}$ & $\mathrm{R}$ & \\
\hline Accipiter erythropus & Epervier de Hartlaub & $\mathrm{V}$ & $\mathrm{U}$ & $\mathrm{LC}$ & $\mathrm{R}$ & GC \\
\hline Accipiter melanoleucus & Autour noir & $\mathrm{V}$ & $\mathrm{F}$ & $\mathrm{LC}$ & $\mathrm{R}$ & \\
\hline Urotriorchis macrourus & Autour à longue queue & $\mathrm{V}$ & $\mathrm{Ra}$ & $\mathrm{LC}$ & $\mathrm{R}$ & GC \\
\hline Kaupifalco monogrammicus & Autour unibande & VAP & $\mathrm{C}$ & $\mathrm{LC}$ & $\mathrm{R}$ & \\
\hline Buteo auguralis & Buse d'Afrique & $\mathrm{V}$ & $\mathrm{F}$ & $\mathrm{LC}$ & $\mathrm{R} / \mathrm{M}$ & \\
\hline Spizaetus africanus & Aigle de Cassin & $\mathrm{V}$ & $\mathrm{Ra}$ & $\mathrm{LC}$ & $\mathrm{R}$ & GC \\
\hline \multicolumn{7}{|l|}{ Falconidae } \\
\hline Falco tinnunculus & Faucon crécerelle & $\mathrm{V}$ & $\mathrm{Ra}$ & $\mathrm{LC}$ & $\mathrm{R} / \mathrm{P}$ & \\
\hline
\end{tabular}


D. B. AHON et al. / Int. J. Biol. Chem. Sci. 6(6): 4031-4045, 2012

\begin{tabular}{|c|c|c|c|c|c|c|}
\hline Falco ardosiaceus & Faucon ardoisé & $\mathrm{V}$ & $\mathrm{F}$ & $\mathrm{LC}$ & $\mathrm{R}$ & \\
\hline Falco cuvierii & Faucon de Cuvier & $\mathrm{V}$ & $\mathrm{F}$ & LC & $\mathrm{R}$ & \\
\hline Falco biarmicus & Faucon lanier & $\mathrm{V}$ & $\mathrm{F}$ & $\mathrm{LC}$ & $\mathrm{R}$ & \\
\hline Falco peregrinus & Faucon pèlerin & VP & $\mathrm{U}$ & $\mathrm{LC}$ & $\mathrm{R} / \mathrm{P}$ & \\
\hline \multicolumn{7}{|l|}{ Phasianidae } \\
\hline Francolinus lathami & Francolin de Latham & $\mathrm{V}$ & $\mathrm{U}$ & LC & $\mathrm{R}$ & GC \\
\hline Francolinus ahantensis & Francolin d'Ahanta & VA & $\mathrm{U}$ & LC & $\mathrm{R}$ & GC \\
\hline Francolinus bicalcaratus & Francolin à double éperon & $\mathrm{V}$ & $\mathrm{U}$ & $\mathrm{LC}$ & $\mathrm{R}$ & \\
\hline \multicolumn{7}{|l|}{ Numididae } \\
\hline Guttera pucherani & Pintade huppée & VA & $\mathrm{U}$ & $\mathrm{LC}$ & $\mathrm{R}$ & \\
\hline \multicolumn{7}{|l|}{ Rallidae } \\
\hline Himantornis haematopus & Râle à pieds rouges & $\mathrm{AE}$ & $\mathrm{C}$ & $\mathrm{LC}$ & $\mathrm{R}$ & GC \\
\hline Sarothrura pulchra & Râle perlé & A & $\mathrm{U}$ & LC & $\mathrm{R}$ & GC \\
\hline Amaurornis flavirostra & Râle à bec jaune & $\mathrm{V}$ & $\mathrm{F}$ & LC & $\mathrm{R}$ & \\
\hline \multicolumn{7}{|l|}{ Heliornithidae } \\
\hline Podica senegalensis & Grébifoulque d'Afrique & $\mathrm{V}$ & $\mathrm{U}$ & LC & $\mathrm{R}$ & \\
\hline \multicolumn{7}{|l|}{ Jacanidae } \\
\hline Actophilornis africanus & Jacana à poitrine dorée & $\mathrm{V}$ & $\mathrm{F}$ & $\mathrm{LC}$ & $\mathrm{R}$ & \\
\hline Microparra capensis & Jacana nain & $\mathrm{V}$ & $\mathrm{F}$ & LC & M & \\
\hline \multicolumn{7}{|l|}{ Burhinidae } \\
\hline Burhinus senegalensis & Oedicnème du Sénégal & $\mathrm{V}$ & $\mathrm{U}$ & $\mathrm{LC}$ & $\mathrm{R}$ & \\
\hline Burhinus vermiculatus & Oedicnème vermiculé & $\mathrm{V}$ & $\mathrm{U}$ & LC & $\mathrm{R}$ & \\
\hline \multicolumn{7}{|l|}{ Charadriidae } \\
\hline Pluvialis fulva & Pluvier fauve & $\mathrm{V}$ & $\mathrm{U}$ & $\mathrm{LC}$ & $\mathrm{O}$ & \\
\hline Vanellus albiceps & Vanneau à tête blanche & $\mathrm{V}$ & $\mathrm{F}$ & $\mathrm{LC}$ & $\mathrm{R}$ & \\
\hline Vanellus spinosus & Vanneau à éperons & $\mathrm{V}$ & $\mathrm{F}$ & $\mathrm{LC}$ & M & \\
\hline \multicolumn{7}{|l|}{ Scolopacidae } \\
\hline Numenius phaeopus & Courlis corlieu & $\mathrm{V}$ & $\mathrm{F}$ & $\mathrm{LC}$ & $\mathrm{P}$ & \\
\hline Tringa nebularia & Chevalier aboyeur & $\mathrm{V}$ & $\mathrm{U}$ & $\mathrm{LC}$ & $\mathrm{P}$ & \\
\hline Tringa ochropus & Chevalier cul-blanc & $\mathrm{V}$ & $\mathrm{U}$ & LC & $\mathrm{P}$ & \\
\hline Actitis hypoleucos & Chevalier guignette & $\mathrm{V}$ & $\mathrm{C}$ & $\mathrm{LC}$ & $\mathrm{P}$ & \\
\hline \multicolumn{7}{|l|}{ Sternidae } \\
\hline Sterna maxima & Sterne royale & $\mathrm{V}$ & $\mathrm{U}$ & LC & $\mathrm{R} / \mathrm{M}$ & \\
\hline Sterna dougallii & Sterne de Dougall & $\mathrm{V}$ & $\mathrm{U}$ & $\mathrm{LC}$ & $\mathrm{P}$ & \\
\hline Sterna hirundo & Sterne pierregarin & $\mathrm{V}$ & $\mathrm{F}$ & LC & $\mathrm{P}$ & \\
\hline \multicolumn{7}{|l|}{ Columbidae } \\
\hline Treron calvus & Colombar à front nu & VAE & $\mathrm{C}$ & LC & $\mathrm{R}$ & \\
\hline Turtur brehmeri & Tourtelette demoiselle & A & $\mathrm{C}$ & $\mathrm{LC}$ & $\mathrm{R}$ & GC \\
\hline Turtur tympanistria & Tourtelette tambourette & VAE & $\mathrm{C}$ & $\mathrm{LC}$ & $\mathrm{R}$ & \\
\hline Turtur afer & Tourtelette améthystine & VA & $\mathrm{C}$ & $\mathrm{LC}$ & $\mathrm{R}$ & \\
\hline Columba iriditorques & Pigeon à nuque bronzée & A & $\mathrm{U}$ & $\mathrm{LC}$ & $\mathrm{R}$ & GC \\
\hline Columba unicincta & Pigeon gris & A & $\mathrm{Ra}$ & $\mathrm{LC}$ & $\mathrm{R}$ & $\mathrm{GC}$ \\
\hline Streptopelia semitorquata & Tourterelle à collier & VAE & $\mathrm{C}$ & $\mathrm{LC}$ & $\mathrm{R}$ & \\
\hline Streptopelia senegalensis & Tourterelle maillée & VAP & $\mathrm{C}$ & $\mathrm{LC}$ & $\mathrm{R}$ & \\
\hline \multicolumn{7}{|l|}{ Psittacidae } \\
\hline Psittacus erithacus & Perroquet jaco & VA & $\mathrm{C}$ & NT & $\mathrm{R}$ & GC \\
\hline Poicephalus gulielmi & Perroquet à calotte rouge & $\mathrm{V}$ & $\mathrm{F}$ & LC & $\mathrm{R}$ & \\
\hline \multicolumn{7}{|l|}{ Musophagidae } \\
\hline Corythaeola cristata & Touraco géant & VA & $\mathrm{U}$ & $\mathrm{LC}$ & $\mathrm{R}$ & \\
\hline Tauraco persa & Touraco vert & $\mathrm{V}$ & $\mathrm{C}$ & $\mathrm{LC}$ & $\mathrm{R}$ & GC \\
\hline Tauraco macrorhynchus & Touraco à gros bec & VAE & $\mathrm{C}$ & $\mathrm{LC}$ & $\mathrm{R}$ & GC \\
\hline Crinifer piscator & Touraco gris & VAP & $\mathrm{U}$ & LC & $\mathrm{R}$ & \\
\hline \multicolumn{7}{|l|}{ Cuculidae } \\
\hline Oxylophus jacobinus & Coucou jacobin & A & $\mathrm{U}$ & $\mathrm{LC}$ & M & \\
\hline
\end{tabular}




\begin{tabular}{|c|c|c|c|c|c|c|c|}
\hline Oxylophus levaillantii & Coucou de Levaillant & VAE & $\mathrm{F}$ & $\mathrm{LC}$ & M & & \\
\hline Pachycoccyx audeberti & Coucou d'Audebert & $\mathrm{V}$ & $\mathrm{Ra}$ & $\mathrm{LC}$ & M & & \\
\hline Cuculus solitarius & Coucou solitaire & VAE & $\mathrm{F}$ & $\mathrm{LC}$ & M & & \\
\hline Cuculus clamosus & Coucou criard & A & $\mathrm{F}$ & $\mathrm{LC}$ & M & & \\
\hline Cuculus gularis & Coucou africain & $\mathrm{V}$ & $\mathrm{U}$ & $\mathrm{LC}$ & M & & \\
\hline Cercococcyx mechowi & Coucou de Mechow & VA & $\mathrm{U}$ & $\mathrm{LC}$ & $\mathrm{R}$ & & GC \\
\hline Cercococcyx olivinus & Coucou olivâtre & VA & $\mathrm{F}$ & $\mathrm{LC}$ & $\mathrm{R}$ & & GC \\
\hline Chrysococcyx cupreus & Coucou foliotocol & VAE & $\mathrm{C}$ & $\mathrm{LC}$ & $\mathrm{R}$ & & \\
\hline Chrysococcyx klaas & Coucou de Klaas & VAE & $\mathrm{C}$ & $\mathrm{LC}$ & $\mathrm{R} / \mathrm{M}$ & & \\
\hline Chrysococcyx caprius & Coucou didric & VAE & $\mathrm{F}$ & $\mathrm{LC}$ & $\mathrm{R} / \mathrm{M}$ & & \\
\hline Ceuthmochares aereus & Malcoha à bec jaune & VAE & $\mathrm{F}$ & $\mathrm{LC}$ & $\mathrm{R}$ & & \\
\hline Centropus leucogaster & Coucal à ventre blanc & VAP & $\mathrm{C}$ & $\mathrm{LC}$ & $\mathrm{R}$ & & GC \\
\hline Centropus grillii & Coucal de Grill & $\mathrm{V}$ & $\mathrm{U}$ & $\mathrm{LC}$ & $\mathrm{M} / \mathrm{R}$ & & \\
\hline Centropus senegalensis & Coucal de Sénégal & VAE & $\mathrm{C}$ & $\mathrm{LC}$ & $\mathrm{R}$ & & \\
\hline \multicolumn{8}{|l|}{ Tytonidae } \\
\hline Tyto alba & Effraie des clochers & VAP & $\mathrm{F}$ & $\mathrm{LC}$ & $\mathrm{R}$ & & \\
\hline \multicolumn{8}{|l|}{ Strigidae } \\
\hline Otus icterorhynchus & Petit-duc à bec jaune & $\mathrm{V}$ & $\mathrm{U}$ & $\mathrm{LC}$ & $\mathrm{R}$ & & GC \\
\hline Ptilopsis leucotis & Petit-duc à face blanche & $\mathrm{V}$ & $\mathrm{Ra}$ & $\mathrm{LC}$ & $\mathrm{R}$ & & \\
\hline Bubo poensis & Grand-duc à aigrettes & VA & $\mathrm{C}$ & $\mathrm{LC}$ & $\mathrm{R}$ & & GC \\
\hline Bubo leucostictus & Grand-duc tacheté & $\mathrm{A}$ & $\mathrm{F}$ & $\mathrm{LC}$ & $\mathrm{R}$ & & GC \\
\hline Scotopelia peli & Chouette-pêcheuse de Pel & A & $\mathrm{Ra}$ & $\mathrm{LC}$ & $\mathrm{R}$ & & \\
\hline Scotopelia ussheri & Chouette-pêcheuse rousse & A & $\mathrm{U}$ & EN & $\mathrm{R}$ & $\mathrm{AO}$ & GC \\
\hline Scotopelia bouvieri & Chouette-pêcheuse de Bouvier & $\mathrm{AE}$ & $\mathrm{U}$ & $\mathrm{LC}$ & $\mathrm{R}$ & & \\
\hline Glaucidium tephronotum & Chevêchette à pieds jaunes & A & $\mathrm{U}$ & $\mathrm{LC}$ & $\mathrm{R}$ & & GC \\
\hline Strix woodfordii & Chouette africaine & VAE & $\mathrm{C}$ & $\mathrm{LC}$ & $\mathrm{R}$ & & \\
\hline \multicolumn{8}{|l|}{ Caprimulgidae } \\
\hline Veles binotatus & Engoulevent à deux taches & VA & $\mathrm{U}$ & $\mathrm{LC}$ & $\mathrm{R}$ & & GC \\
\hline Caprimulgus climacurus & Engoulevent à longue queue & VA & $\mathrm{C}$ & $\mathrm{LC}$ & $\mathrm{R} / \mathrm{M}$ & & \\
\hline \multicolumn{8}{|l|}{ Apodidae } \\
\hline Rhaphidura sabini & Martinet de Sabine & $\mathrm{V}$ & $\mathrm{C}$ & $\mathrm{LC}$ & $\mathrm{R}$ & & GC \\
\hline Telacanthura melanopygia & Martinet de Chapin & $\mathrm{V}$ & $\mathrm{C}$ & $\mathrm{LC}$ & $\mathrm{R}$ & & GC \\
\hline Telacanthura ussheri & Martinet d'Ussher & $\mathrm{V}$ & $\mathrm{C}$ & $\mathrm{LC}$ & $\mathrm{R}$ & & \\
\hline Cypsiurus parvus & Martinet des palmiers & $\mathrm{V}$ & $\mathrm{C}$ & $\mathrm{LC}$ & $\mathrm{R}$ & & \\
\hline Apus apus & Martinet noir & $\mathrm{V}$ & $\mathrm{C}$ & $\mathrm{LC}$ & $\mathrm{P}$ & & \\
\hline Apus affinis & Martinet des maisons & $\mathrm{V}$ & $\mathrm{C}$ & $\mathrm{LC}$ & $\mathrm{R}$ & & \\
\hline \multicolumn{8}{|l|}{ Trogonidae } \\
\hline Apaloderma narina & Trogon narina & VA & $\mathrm{U}$ & $\mathrm{LC}$ & $\mathrm{R}$ & & \\
\hline \multicolumn{8}{|l|}{ Alcedinidae } \\
\hline Halcyon leucocephala & Martin-chasseur à tête grise & $\mathrm{V}$ & $\mathrm{U}$ & $\mathrm{LC}$ & M & & \\
\hline \multirow[t]{2}{*}{ Halcyon malimbica } & Martin-chasseur à poitrine & & & & & & \\
\hline & bleue & V & $\mathrm{U}$ & $\mathrm{LC}$ & $\mathrm{R}$ & & \\
\hline Halcyon senegalensis & Martin-chasseur du Sénégal & VAP & $\mathrm{C}$ & $\mathrm{LC}$ & $\mathrm{R}$ & & \\
\hline Ceyx lecontei & Martin-pêcheur à tête rousse & $\mathrm{V}$ & $\mathrm{U}$ & $\mathrm{LC}$ & $\mathrm{R}$ & & GC \\
\hline Ceyx pictus & Martin-pêcheur pygmée & $\mathrm{V}$ & $\mathrm{C}$ & $\mathrm{LC}$ & $\mathrm{R}$ & & \\
\hline Alcedo leucogaster & Martin-pêcheur à vent blanc & VPJ & $\mathrm{F}$ & $\mathrm{LC}$ & $\mathrm{R}$ & & GC \\
\hline Alcedo cristata & Martin-pêcheur huppé & $\mathrm{V}$ & $\mathrm{U}$ & $\mathrm{LC}$ & $\mathrm{R}$ & & \\
\hline Ceryle rudis & Martin-pêcheur pie & VP & $\mathrm{F}$ & $\mathrm{LC}$ & $\mathrm{R}$ & & \\
\hline \multicolumn{8}{|l|}{ Meropidae } \\
\hline Merops gularis & Guêpier noir & $\mathrm{V}$ & $\mathrm{C}$ & $\mathrm{LC}$ & $\mathrm{R}$ & & GC \\
\hline Merops pusillus & Guêpier nain & $\mathrm{V}$ & $\mathrm{C}$ & $\mathrm{LC}$ & $\mathrm{R}$ & & \\
\hline Merops albicollis & Guêpier à gorge blanche & $\mathrm{V}$ & $\mathrm{C}$ & $\mathrm{LC}$ & M & & \\
\hline \multicolumn{8}{|l|}{ Coraciidae } \\
\hline Coracias cyanogaster & Rollier à ventre bleu & $\mathrm{V}$ & $\mathrm{Ra}$ & $\mathrm{LC}$ & $\mathrm{R}$ & & SG \\
\hline Coracias abyssinicus & Rollier d'Abyssinie & $\mathrm{V}$ & $\mathrm{U}$ & $\mathrm{LC}$ & M & & \\
\hline
\end{tabular}


D. B. AHON et al. / Int. J. Biol. Chem. Sci. 6(6): 4031-4045, 2012

\begin{tabular}{|c|c|c|c|c|c|c|c|}
\hline $\begin{array}{l}\text { Eurystomus gularis } \\
\text { Eurystomus glaucurus }\end{array}$ & $\begin{array}{l}\text { Rolle à gorge bleue } \\
\text { Rolle violet }\end{array}$ & $\begin{array}{l}\mathrm{V} \\
\mathrm{V}\end{array}$ & $\begin{array}{l}\mathrm{F} \\
\mathrm{C}\end{array}$ & $\begin{array}{l}\mathrm{LC} \\
\mathrm{LC}\end{array}$ & $\begin{array}{c}\mathrm{R} \\
\mathrm{R} / \mathrm{M}\end{array}$ & & GC \\
\hline \multicolumn{8}{|l|}{ Phoeniculidae } \\
\hline Rhinopomastus aterrimus & Irrisor noir & $\mathrm{V}$ & $\mathrm{F}$ & $\mathrm{LC}$ & $\mathrm{R}$ & & \\
\hline \multicolumn{8}{|l|}{ Bucerotidae } \\
\hline Tropicranus albocristatus & Calao à huppe blanche & VAP & $\mathrm{C}$ & $\mathrm{LC}$ & $\mathrm{R}$ & & GC \\
\hline Tockus fasciatus & Calao longibande & VAP & $\mathrm{C}$ & $\mathrm{LC}$ & $\mathrm{R}$ & & GC \\
\hline Bycanistes fistulator & Calao siffleur & VAP & $\mathrm{C}$ & $\mathrm{LC}$ & $\mathrm{R}$ & & GC \\
\hline Ceratogymna atrata & Calao à casque noir & $\mathrm{V}$ & $\mathrm{U}$ & $\mathrm{LC}$ & $\mathrm{R}$ & & $\mathrm{GC}$ \\
\hline Ceratogymna elata & Calao à casque jaune & A & $\mathrm{U}$ & NT & $\mathrm{R}$ & $\mathrm{AO}$ & GC \\
\hline \multicolumn{8}{|l|}{ Capitonidae } \\
\hline Gymnobucco peli & Barbican à narines emplumées & $\mathrm{A}$ & $\mathrm{F}$ & $\mathrm{LC}$ & $\mathrm{R}$ & & GC \\
\hline Gymnobucco calvus & Barbican chauve & $\mathrm{A}$ & $\mathrm{C}$ & $\mathrm{LC}$ & $\mathrm{R}$ & & GC \\
\hline Pogoniulus scolopaceus & Barbion grivelé & A & $\mathrm{C}$ & $\mathrm{LC}$ & $\mathrm{R}$ & & GC \\
\hline Pogoniulus atroflavus & Barbion à croupion rouge & A & $\mathrm{C}$ & $\mathrm{LC}$ & $\mathrm{R}$ & & $\mathrm{GC}$ \\
\hline Pogoniulus subsulphureus & Barbion à gorge jaune & VA & $\mathrm{C}$ & $\mathrm{LC}$ & $\mathrm{R}$ & & GC \\
\hline Pogoniulus bilineatus & Barbion à croupion jaune & VA & $\mathrm{U}$ & $\mathrm{LC}$ & $\mathrm{R}$ & & \\
\hline Buccanodon duchaillui & Barbican à taches jaunes & A & $\mathrm{F}$ & $\mathrm{LC}$ & $\mathrm{R}$ & & $\mathrm{GC}$ \\
\hline Tricholaema hirsuta & Barbican hérissé & A & $\mathrm{F}$ & $\mathrm{LC}$ & $\mathrm{R}$ & & GC \\
\hline Lybius vieilloti & Barbican de Vieillot & $\mathrm{V}$ & $\mathrm{Ra}$ & $\mathrm{LC}$ & $\mathrm{R}$ & & \\
\hline Trachylaemus purpuratus & Barbican pourpré & A & $\mathrm{F}$ & $\mathrm{LC}$ & $\mathrm{R}$ & & GC \\
\hline \multicolumn{8}{|l|}{ Indicatoridae } \\
\hline Prodotiscus insignis & Indicateur pygmée & $\mathrm{V}$ & $\mathrm{U}$ & $\mathrm{LC}$ & $\mathrm{R}$ & & GC \\
\hline Melignomon eisentrauti & Indicateur d"Eisentraut & $\mathrm{V}$ & $\mathrm{U}$ & DD & $\mathrm{R}$ & $\mathrm{AO}$ & GC \\
\hline Indicator maculatus & Indicateur tacheté & $\mathrm{V}$ & $\mathrm{U}$ & $\mathrm{LC}$ & $\mathrm{R}$ & & GC \\
\hline \multicolumn{8}{|l|}{ Picidae } \\
\hline Campethera maculosa & Pic barré & $\mathrm{V}$ & $\mathrm{U}$ & $\mathrm{LC}$ & $\mathrm{R}$ & & GC \\
\hline Campethera nivosa & Pic tacheté & $\mathrm{V}$ & $\mathrm{F}$ & $\mathrm{LC}$ & $\mathrm{R}$ & & $\mathrm{GC}$ \\
\hline Dendropicos gabonensis & Pic du Gabon & $\mathrm{V}$ & $\mathrm{C}$ & $\mathrm{LC}$ & $\mathrm{R}$ & & GC \\
\hline Dendropicos pyrrhogaster & Pic à ventre de feu & VA & $\mathrm{C}$ & $\mathrm{LC}$ & $\mathrm{R}$ & & GC \\
\hline \multicolumn{8}{|l|}{ Eurylaimidae } \\
\hline Smithornis rufolateralis & Eurylaime à flancs roux & $\mathrm{V}$ & $\mathrm{Ra}$ & $\mathrm{LC}$ & $\mathrm{R}$ & & $\mathrm{GC}$ \\
\hline \multicolumn{8}{|l|}{ Hirundinidae } \\
\hline Hirundo senegalensis & Hirondelle des mosquées & $\mathrm{V}$ & $\mathrm{F}$ & $\mathrm{LC}$ & $\mathrm{R}$ & & \\
\hline Hirundo abyssinica & Hirondelle striée & $\mathrm{V}$ & $\mathrm{C}$ & $\mathrm{LC}$ & $\mathrm{R}$ & & \\
\hline Hirundo daurica & Hirondelle rousseline & $\mathrm{V}$ & $\mathrm{C}$ & $\mathrm{LC}$ & $\mathrm{R}$ & & \\
\hline Hirundo nigrita & Hirondelle à bavette & $\mathrm{V}$ & $\mathrm{C}$ & $\mathrm{LC}$ & $\mathrm{R}$ & & $\mathrm{GC}$ \\
\hline Hirundo rustica & Hirondelle rustique & $\mathrm{V}$ & $\mathrm{C}$ & $\mathrm{LC}$ & $\mathrm{P}$ & & \\
\hline Delichon urbicum & Hirondelle de fenêtre & $\mathrm{V}$ & $\mathrm{F}$ & $\mathrm{LC}$ & $\mathrm{P}$ & & \\
\hline \multicolumn{8}{|l|}{ Motacillidae } \\
\hline Motacilla flava & Bergeronnette printanière & V & $\mathrm{U}$ & $\mathrm{LC}$ & $\mathrm{P}$ & & \\
\hline Motacilla aguimp & Bergeronnette pie & $\mathrm{V}$ & $\mathrm{F}$ & $\mathrm{LC}$ & $\mathrm{R}$ & & \\
\hline Anthus leucophrys & Pipit à dos uni & VP & $\mathrm{C}$ & $\mathrm{LC}$ & $\mathrm{R}$ & & \\
\hline \multicolumn{8}{|l|}{ Campephagidae } \\
\hline Lobotus lobatus & Echenilleur à barbillons & $\mathrm{V}$ & $\mathrm{Ra}$ & $\mathrm{LC}$ & $\mathrm{R}$ & $\mathrm{AO}$ & GC \\
\hline \multicolumn{8}{|l|}{ Pycnonotidae } \\
\hline Andropadus virens & Bulbul verdâtre & VAJ & $\mathrm{C}$ & $\mathrm{LC}$ & $\mathrm{R}$ & & \\
\hline Andropadus gracilis & Bulbul gracile & $\mathrm{V}$ & $\mathrm{C}$ & $\mathrm{LC}$ & $\mathrm{R}$ & & GC \\
\hline Andropadus ansorgei & Bulbul d'Ansorge & $\mathrm{V}$ & $\mathrm{F}$ & $\mathrm{LC}$ & $\mathrm{R}$ & & $\mathrm{GC}$ \\
\hline Andropadus curvirostris & Bulbul curvirostre & $\mathrm{V}$ & $\mathrm{Ra}$ & $\mathrm{LC}$ & $\mathrm{R}$ & & GC \\
\hline Andropadus gracilirostris & Bulbul à bec grêle & $\mathrm{V}$ & $\mathrm{C}$ & $\mathrm{LC}$ & $\mathrm{R}$ & & \\
\hline Andropadus latirostris & Bulbul à moustaches jaunes & VAJ & $\mathrm{C}$ & $\mathrm{LC}$ & $\mathrm{R}$ & & \\
\hline Baeopogon indicator & Bulbul à queue blanche & $\mathrm{V}$ & $\mathrm{F}$ & $\mathrm{LC}$ & $\mathrm{R}$ & & GC \\
\hline Ixonotus guttatus & Bulbul tacheté & $\mathrm{V}$ & $\mathrm{U}$ & $\mathrm{LC}$ & $\mathrm{R}$ & & $\mathrm{GC}$ \\
\hline Chlorocichla simplex & Bulbul modeste & $\mathrm{V}$ & $\mathrm{U}$ & $\mathrm{LC}$ & $\mathrm{R}$ & & GC \\
\hline
\end{tabular}




\begin{tabular}{|c|c|c|c|c|c|c|c|}
\hline Thescelocichla leucopleura & Bulbul des raphias & VA & $\mathrm{C}$ & $\mathrm{LC}$ & $\mathrm{R}$ & & $\mathrm{GC}$ \\
\hline Pyrrhurus scandens & Bulbul à queue rousse & V & $\mathrm{F}$ & LC & $\mathrm{R}$ & & GC \\
\hline Phyllastrephus icterinus & Bulbul icterin & $\mathrm{V}$ & $\mathrm{F}$ & $\mathrm{LC}$ & $\mathrm{R}$ & & GC \\
\hline Bleda syndactylus & Bulbul moustac & VA & $\mathrm{C}$ & LC & $\mathrm{R}$ & & GC \\
\hline Bleda eximius & Bulbul à queue verte & VA & $\mathrm{C}$ & $\mathrm{VU}$ & $\mathrm{R}$ & $\mathrm{AO}$ & GC \\
\hline Bleda canicapillus & Bulbul fourmilier & VA & $\mathrm{C}$ & $\mathrm{LC}$ & $\mathrm{R}$ & & GC \\
\hline Criniger calurus & Bulbul à barbe blanche & $\mathrm{V}$ & $\mathrm{U}$ & $\mathrm{LC}$ & $\mathrm{R}$ & & GC \\
\hline Criniger olivaceus & Bulbul à barbe jaune & $\mathrm{V}$ & $\mathrm{F}$ & VU & $\mathrm{R}$ & $\mathrm{AO}$ & GC \\
\hline Pycnonotus barbatus & Bulbul des jardins & VAP & $\mathrm{C}$ & $\mathrm{LC}$ & $\mathrm{R}$ & & \\
\hline Nicator chloris & Bulbul nicator & $\mathrm{AE}$ & $\mathrm{C}$ & LC & $\mathrm{R}$ & & GC \\
\hline \multicolumn{8}{|l|}{ Turdidae } \\
\hline Stiphrornis erythrothorax & Rougegorge de forêt & $\mathrm{V}$ & $\mathrm{F}$ & LC & $\mathrm{R}$ & & GC \\
\hline Alethe diademata & Alèthe à huppe rousse & VA & $\mathrm{C}$ & $\mathrm{LC}$ & $\mathrm{R}$ & & GC \\
\hline Neocossyphus poensis & Néocossyphe à queue blanche & $\mathrm{V}$ & $\mathrm{F}$ & $\mathrm{LC}$ & $\mathrm{R}$ & & GC \\
\hline Stizorhina finschi & Stizorhin de Finsch & A & $\mathrm{U}$ & $\mathrm{LC}$ & $\mathrm{R}$ & & \\
\hline \multicolumn{8}{|l|}{ Sylviidae } \\
\hline Bathmocercus cerviniventris & Bathmocerque à capuchon & $\mathrm{V}$ & $\mathrm{F}$ & NT & $\mathrm{R}$ & $\mathrm{AO}$ & GC \\
\hline Acrocephalus schoenobaenus & Phragmite des joncs & $\mathrm{V}$ & $\mathrm{C}$ & $\mathrm{LC}$ & $\mathrm{P}$ & & \\
\hline Acrocephalus scirpaceus & Rousserolle effarvatte & $\mathrm{V}$ & $\mathrm{C}$ & $\mathrm{LC}$ & $\mathrm{P}$ & & \\
\hline Acrocephalus arundinaceus & Rousserolle turdoïde & $\mathrm{V}$ & $\mathrm{C}$ & $\mathrm{LC}$ & $\mathrm{P}$ & & \\
\hline Cisticola lateralis & Cisticole siffleuse & $\mathrm{V}$ & $\mathrm{C}$ & $\mathrm{LC}$ & $\mathrm{R}$ & & \\
\hline Cisticola galactotes & Cisticole roussâtre & $\mathrm{V}$ & $\mathrm{C}$ & LC & $\mathrm{R}$ & & \\
\hline Cisticola brachypterus & Cisticole à ailes courtes & $\mathrm{V}$ & $\mathrm{F}$ & $\mathrm{LC}$ & $\mathrm{R}$ & & \\
\hline Prinia subflava & Prinia modeste & $\mathrm{V}$ & $\mathrm{C}$ & $\mathrm{LC}$ & $\mathrm{R}$ & & \\
\hline Apalis nigriceps & Apalis à calotte noire & $\mathrm{V}$ & $\mathrm{U}$ & LC & $\mathrm{R}$ & & \\
\hline Apalis sharpii & Apalis de Sharpe & VA & $\mathrm{C}$ & $\mathrm{LC}$ & $\mathrm{R}$ & $\mathrm{AO}$ & GC \\
\hline Camaroptera brachyura & Camaroptère à tête grise & VA & $\mathrm{C}$ & $\mathrm{LC}$ & $\mathrm{R}$ & & \\
\hline Camaroptera superciliaris & Camaroptère à sourcils jaunes & $\mathrm{V}$ & $\mathrm{F}$ & $\mathrm{LC}$ & $\mathrm{R}$ & & GC \\
\hline Camaroptera chloronota & Camaroptère à dos vert & $\mathrm{V}$ & $\mathrm{F}$ & $\mathrm{LC}$ & $\mathrm{R}$ & & GC \\
\hline Sylvietta virens & Crombec vert & VA & $\mathrm{C}$ & $\mathrm{LC}$ & $\mathrm{R}$ & & GC \\
\hline Hypergerus atriceps & Noircap loriot & $\mathrm{V}$ & $\mathrm{U}$ & $\mathrm{LC}$ & $\mathrm{R}$ & $\mathrm{AO}$ & SG \\
\hline Sylvia borin & Fauvette des jardins & $\mathrm{V}$ & $\mathrm{C}$ & $\mathrm{LC}$ & $\mathrm{P}$ & & \\
\hline Hyliota violacea & Hyliote à dos violet & $\mathrm{V}$ & $\mathrm{C}$ & $\mathrm{LC}$ & $\mathrm{R}$ & & GC \\
\hline Hylia prasina & Hylia verte & VAJ & $\mathrm{C}$ & $\mathrm{LC}$ & $\mathrm{R}$ & & GC \\
\hline \multicolumn{8}{|l|}{ Muscicapidae } \\
\hline Fraseria ocreata & Gobemouche forestier & $\mathrm{V}$ & $\mathrm{F}$ & $\mathrm{LC}$ & $\mathrm{R}$ & & GC \\
\hline Muscicapa striata & Gobemouche gris & $\mathrm{V}$ & $\mathrm{C}$ & $\mathrm{LC}$ & $\mathrm{R}$ & & \\
\hline Muscicapa caerulescens & Gobemouche à lunettes & $\mathrm{V}$ & $\mathrm{C}$ & $\mathrm{LC}$ & $\mathrm{R}$ & & \\
\hline Muscicapa cassini & Gobemouche de Cassin & $\mathrm{V}$ & $\mathrm{C}$ & $\mathrm{LC}$ & $\mathrm{R}$ & & GC \\
\hline Muscicapa olivascens & Gobemouche olivâtre & $\mathrm{V}$ & $\mathrm{F}$ & $\mathrm{LC}$ & $\mathrm{R}$ & & GC \\
\hline Muscicapa comitata & Gobemouche ardoisé & $\mathrm{V}$ & $\mathrm{C}$ & $\mathrm{LC}$ & $\mathrm{R}$ & & GC \\
\hline Muscicapa ussheri & Gobemouche d'Ussher & $\mathrm{V}$ & $\mathrm{C}$ & $\mathrm{LC}$ & $\mathrm{R}$ & $\mathrm{AO}$ & GC \\
\hline Ficedula hypoleuca & Gobemouche noir & $\mathrm{V}$ & $\mathrm{C}$ & $\mathrm{LC}$ & $\mathrm{P}$ & & \\
\hline \multicolumn{8}{|l|}{ Monarchidae } \\
\hline Terpsiphone viridis & Tchitrec d'Afrique & $\mathrm{V}$ & $\mathrm{F}$ & $\mathrm{LC}$ & $\mathrm{R} / \mathrm{M}$ & & \\
\hline Terpsiphone rufiventer & Tchitrec à ventre roux & VA & $\mathrm{C}$ & $\mathrm{LC}$ & $\mathrm{R}$ & & GC \\
\hline \multicolumn{8}{|l|}{ Platysteiridae } \\
\hline Dyaphorophyia castanea & Pririt châtain & $\mathrm{V}$ & $\mathrm{C}$ & $\mathrm{LC}$ & $\mathrm{R}$ & & GC \\
\hline Dyaphorophyia concreta & Pririt à ventre doré & $\mathrm{V}$ & $\mathrm{C}$ & $\mathrm{LC}$ & $\mathrm{R}$ & & \\
\hline Platysteira cyanea & Pririt à collier & V & $\mathrm{C}$ & $\mathrm{LC}$ & $\mathrm{R}$ & & \\
\hline \multicolumn{8}{|l|}{ Timaliidae } \\
\hline Illadopsis fulvescens & Akalat brun & $\mathrm{V}$ & $\mathrm{C}$ & LC & $\mathrm{R}$ & & GC \\
\hline Illadopsis rufescens & Akalat à ailes rousses & $\mathrm{V}$ & $\mathrm{U}$ & NT & $\mathrm{R}$ & $\mathrm{AO}$ & GC \\
\hline \multicolumn{8}{|l|}{ Nectariniidae } \\
\hline Anthreptes gabonicus & Souimanga brun & $\mathrm{V}$ & $\mathrm{F}$ & $\mathrm{LC}$ & $\mathrm{R}$ & & GC \\
\hline
\end{tabular}


D. B. AHON et al. / Int. J. Biol. Chem. Sci. 6(6): 4031-4045, 2012

\begin{tabular}{|c|c|c|c|c|c|c|c|}
\hline Anthreptes rectirostris & Souimanga à bec droit & $\mathrm{V}$ & $\mathrm{C}$ & $\mathrm{LC}$ & $\mathrm{R}$ & & GC \\
\hline Deleornis fraseri & Souimanga de Fraser & $\mathrm{V}$ & $\mathrm{C}$ & $\mathrm{LC}$ & $\mathrm{R}$ & & GC \\
\hline Anabathmis reichenbachii & Souimanga de Reichenbach & VAJ & $\mathrm{C}$ & LC & $\mathrm{R}$ & & GC \\
\hline Cyanomitra verticalis & Souimanga à tête verte & $\mathrm{V}$ & $\mathrm{F}$ & LC & $\mathrm{R}$ & & \\
\hline Cyanomitra obscura & Souimanga olivâtre & VJ & $\mathrm{C}$ & $\mathrm{LC}$ & $\mathrm{R}$ & & \\
\hline Chalcomitra adelberti & Souimanga à gorge rousse & V & $\mathrm{C}$ & LC & $\mathrm{R}$ & $\mathrm{AO}$ & GC \\
\hline Chalcomitra fuliginosa & Souimanga carmélite & $\mathrm{V}$ & $\mathrm{F}$ & LC & $\mathrm{R}$ & & GC \\
\hline Hedydipna collaris & Souimanga à collier & VA & $\mathrm{C}$ & LC & $\mathrm{R}$ & & \\
\hline Cinnyris chloropygius & Souimanga à ventre olive & $\mathrm{V}$ & $\mathrm{C}$ & LC & $\mathrm{R}$ & & \\
\hline Cinnyris johannae & Souimanga de Johanna & $\mathrm{V}$ & $\mathrm{F}$ & LC & $\mathrm{R}$ & & GC \\
\hline Cinnyris superbus & Souimanga superbe & $\mathrm{V}$ & $\mathrm{C}$ & LC & $\mathrm{R}$ & & GC \\
\hline Cinnyris coccinigastrus & Souimanga éclatant & VJ & $\mathrm{U}$ & LC & $\mathrm{R}$ & & SG \\
\hline Cinnyris cupreus & Souimanga cuivré & $\mathrm{V}$ & $\mathrm{F}$ & $\mathrm{LC}$ & $\mathrm{R}$ & & \\
\hline \multicolumn{8}{|l|}{ Laniidae } \\
\hline Lanius collaris & Pie-grièche fiscale & V & $\mathrm{F}$ & LC & $\mathrm{R}$ & & \\
\hline \multicolumn{8}{|l|}{ Malaconotidae } \\
\hline Tchagra australis & Tchagra à tête brune & $\mathrm{V}$ & $\mathrm{F}$ & $\mathrm{LC}$ & $\mathrm{R}$ & & \\
\hline Tchagra senegalus & Tchagra à tête noire & VA & $\mathrm{C}$ & LC & $\mathrm{R}$ & & \\
\hline Laniarius leucorhynchus & Gonolek fuligineux & VA & $\mathrm{U}$ & $\mathrm{LC}$ & $\mathrm{R}$ & & \\
\hline Laniarius barbarus & Gonolek de Barbarie & VA & $\mathrm{Ra}$ & LC & $\mathrm{R}$ & $\mathrm{AO}$ & \\
\hline \multicolumn{8}{|l|}{ Prionopidae } \\
\hline Prionops caniceps & Bagadais à bec rouge & $\mathrm{V}$ & $\mathrm{F}$ & $\mathrm{LC}$ & $\mathrm{R}$ & & \\
\hline \multicolumn{8}{|l|}{ Oriolidae } \\
\hline Oriolus nigripennis & Loriot à ailes noires & $\mathrm{V}$ & $\mathrm{C}$ & $\mathrm{LC}$ & $\mathrm{R}$ & & GC \\
\hline Oriolus brachyrhynchus & Loriot à tête noire & VA & $\mathrm{C}$ & LC & $\mathrm{R}$ & & GC \\
\hline \multicolumn{8}{|l|}{ Dicruridae } \\
\hline Dicrurus atripennis & Drongo de forêt & $\mathrm{V}$ & $\mathrm{F}$ & LC & $\mathrm{R}$ & & GC \\
\hline Dicrurus modestus & Drongo modeste & $\mathrm{V}$ & $\mathrm{F}$ & LC & $\mathrm{R}$ & & \\
\hline \multicolumn{8}{|l|}{ Corvidae } \\
\hline Corvus albus & Corbeau pie & VAP & $\mathrm{C}$ & $\mathrm{LC}$ & $\mathrm{R}$ & & \\
\hline \multicolumn{8}{|l|}{ Sturnidae } \\
\hline Onychognathus fulgidus & Rufipenne de forêt & $\mathrm{V}$ & $\mathrm{F}$ & $\mathrm{LC}$ & $\mathrm{R}$ & & \\
\hline Lamprotornis cupreocauda & Choucador à queue bronzée & $\mathrm{V}$ & $\mathrm{F}$ & NT & $\mathrm{R}$ & $\mathrm{AO}$ & GC \\
\hline Lamprotornis purpureus & Choucador pourprée & $\mathrm{V}$ & $\mathrm{F}$ & $\mathrm{LC}$ & $\mathrm{R}$ & & SG \\
\hline Lamprotornis splendidus & Choucador splendide & VP & $\mathrm{C}$ & $\mathrm{LC}$ & $\mathrm{R}$ & & \\
\hline Cinnyricinclus leucogaster & Spréo améthyste & $\mathrm{V}$ & $\mathrm{C}$ & LC & M & & \\
\hline \multicolumn{8}{|l|}{ Passeridae } \\
\hline Passer griseus & Moineau gris & VAP & $\mathrm{C}$ & $\mathrm{LC}$ & $\mathrm{R}$ & & \\
\hline \multicolumn{8}{|l|}{ Ploceidae } \\
\hline Malimbus scutatus & Malimbe à queue rouge & $\mathrm{V}$ & $\mathrm{C}$ & LC & $\mathrm{R}$ & $\mathrm{AO}$ & GC \\
\hline Malimbus malimbicus & Malimbe huppé & $\mathrm{V}$ & $\mathrm{U}$ & $\mathrm{LC}$ & $\mathrm{R}$ & & GC \\
\hline Malimbus nitens & Malimbe à bec bleu & $\mathrm{V}$ & $\mathrm{U}$ & $\mathrm{LC}$ & $\mathrm{R}$ & & GC \\
\hline Malimbus rubricollis & Malimbe à tête rouge & $\mathrm{V}$ & $\mathrm{F}$ & $\mathrm{LC}$ & $\mathrm{R}$ & & $\mathrm{GC}$ \\
\hline Ploceus nigricollis & Tisserin à cou noir & $\mathrm{V}$ & $\mathrm{F}$ & $\mathrm{LC}$ & $\mathrm{R}$ & & \\
\hline Ploceus aurantius & Tisserin orangé & $\mathrm{V}$ & $\mathrm{C}$ & $\mathrm{LC}$ & $\mathrm{R}$ & & GC \\
\hline Ploceus nigerrimus & Tisserin noir & $\mathrm{V}$ & $\mathrm{C}$ & $\mathrm{LC}$ & $\mathrm{R}$ & & GC \\
\hline Ploceus cucullatus & Tisserin gendarme & VAP & $\mathrm{C}$ & $\mathrm{LC}$ & $\mathrm{R}$ & & \\
\hline Ploceus tricolor & Tisserin tricolore & $\mathrm{V}$ & $\mathrm{F}$ & $\mathrm{LC}$ & $\mathrm{R}$ & & GC \\
\hline Euplectes hordeaceus & Euplecte monseigneur & $\mathrm{V}$ & $\mathrm{F}$ & $\mathrm{LC}$ & $\mathrm{R}$ & & \\
\hline Euplectes afer & Euplecte vorabé & $\mathrm{V}$ & $\mathrm{F}$ & $\mathrm{LC}$ & $\mathrm{R}$ & & \\
\hline \multicolumn{8}{|l|}{ Estrildidae } \\
\hline Nigrita canicapillus & Nigrette à calotte grise & VAE & $\mathrm{C}$ & $\mathrm{LC}$ & $\mathrm{R}$ & & \\
\hline Nigrita bicolor & Nigrette à ventre roux & $\mathrm{V}$ & $\mathrm{C}$ & $\mathrm{LC}$ & $\mathrm{R}$ & & GC \\
\hline Nigrita fusconotus & Nigrette à ventre blanc & $\mathrm{V}$ & $\mathrm{F}$ & $\mathrm{LC}$ & $\mathrm{R}$ & & GC \\
\hline Estrilda melpoda & Astrild à joues oranges & $\mathrm{V}$ & $\mathrm{C}$ & LC & $\mathrm{R}$ & & \\
\hline
\end{tabular}




\begin{tabular}{|c|c|c|c|c|c|c|}
\hline Spermophaga haematina & Sénégali sanguin & VP & $\mathrm{F}$ & LC & $\mathrm{R}$ & GC \\
\hline Pyrenestes sanguineus & Pyréneste gros-bec & V & $\mathrm{F}$ & LC & $\mathrm{R}$ & $\mathrm{GC}$ \\
\hline Spermestes cucullatus & Capucin nonnette & VP & $\mathrm{C}$ & LC & R & \\
\hline Spermestes bicolor & Capucin bicolore & V & C & LC & $\mathrm{R}$ & \\
\hline Spermestes fringilloides & Capucin pie & V & $\mathrm{F}$ & LC & $\mathrm{R}$ & \\
\hline \multicolumn{7}{|l|}{ Viduidae } \\
\hline Vidua macroura & Veuve dominicaine & VP & $\mathrm{C}$ & LC & $\mathrm{R}$ & \\
\hline \multicolumn{7}{|c|}{ 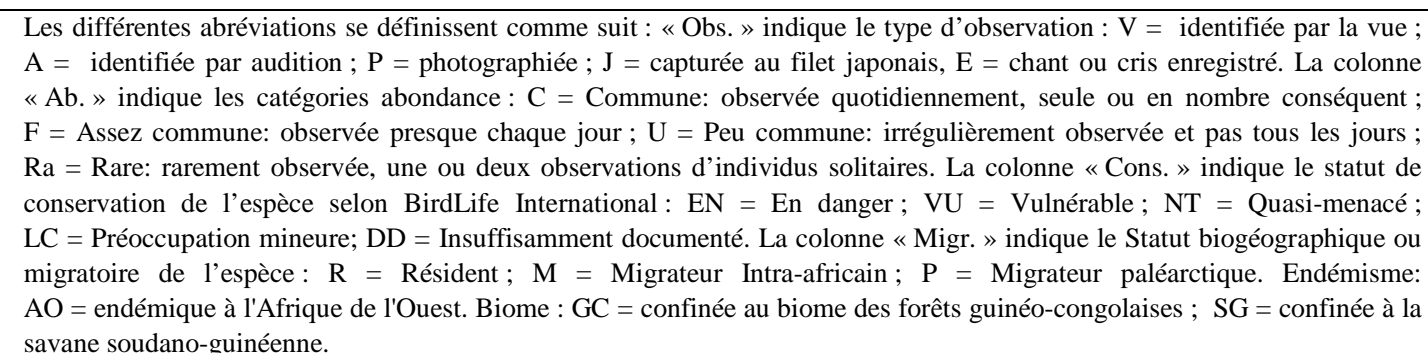 } \\
\hline
\end{tabular}

\section{DISCUSSION}

L'importance numérique des oiseaux de la FMTE montre l'intérêt de cet écosystème forestier pour les oiseaux. Ces 274 espèces représentent $36,24 \%$ de l'ensemble des espèces d'oiseaux rencontrées en Côte d'Ivoire qui est de 756 (Halle et Bruzon 2006). Cet effectif est relativement élevé compte tenu de la courte période d'étude sur le terrain et en comparaison aux travaux de Kouadio (2006), dans la Forêt Classée de N'ganda-N'ganda (zone marécageuse du sudest de la Côte d'Ivoire) où 95 espèces d'oiseaux ont été recensées en deux mois.

Avec douze espèces d'oiseaux dont la protection est d'intérêt mondial (BirdLife International, 2010) parmi lesquelles cinq (espèces listées comme étant en danger et vulnérables) sont inscrites sur la Liste Rouge des espèces d'oiseaux menacées, la FMTE mérite une attention particulière car d'autres espèces pourraient être recensées dans les études ultérieures. En effet, excepté le Parc National de Taï et la Réserve de faune du N'zo (ZICO CI 011), la FMTE avec un nombre de huit espèces renferme plus d'espèces à répartition restreinte que les 13 autres ZICO confirmées de la Côte d'Ivoire.

La proportion de $17,51 \%$ d'espèces migratrices parmi les espèces recensées apparaît être assez représentative et très intéressante, étant donné que le pic de la migration des oiseaux se situe autour du mois de janvier en Côte d'Ivoire (Thiollay 1985). Il est presque certain que d'autres espèces d'oiseaux migrateurs auraient été observées dans cette période. Huit des 14 espèces à répartition restreinte (soit $57,14 \%$ ) recensées en Côte d'Ivoire (Fishpool, 2001; Stattersfield et al., 1998) ont été trouvées dans la FMTE. Ce qui semble être assez représentatif pour cette forêt très peu étendue (en comparaison au Parc National de Taï) et ne possédant pas de statut légal de protection à l'état actuel (Béné et al., 2012). Aussi, avec 110 des 185 (soit 59,46\%) espèces d'oiseaux confinées au biome de la forêt Guinéo-Congolaise, la FMTE renferme plus d'espèces que le Parc National d'Azagny (ZICO CI 014) dont la physionomie des habitats lui est semblable. En effet, ce parc ne renferme que 80 espèces d'oiseaux confinées au biome des forêts Guinéo-Congolaises (Lauginie, 2007). Seulement cinq sites en Côte d'Ivoire, classés comme ZICO, ont un nombre plus élevé d'espèces inféodées à un biome que la FMTE : le PN de Taï (157 espèces), le PN du Mont Péko (144), la Forêt de Yapo (135), le PN de la Marahoué (117 espèces) et la Forêt de Classée de Mopri (115 espèces) (Fishpool, 2001). 


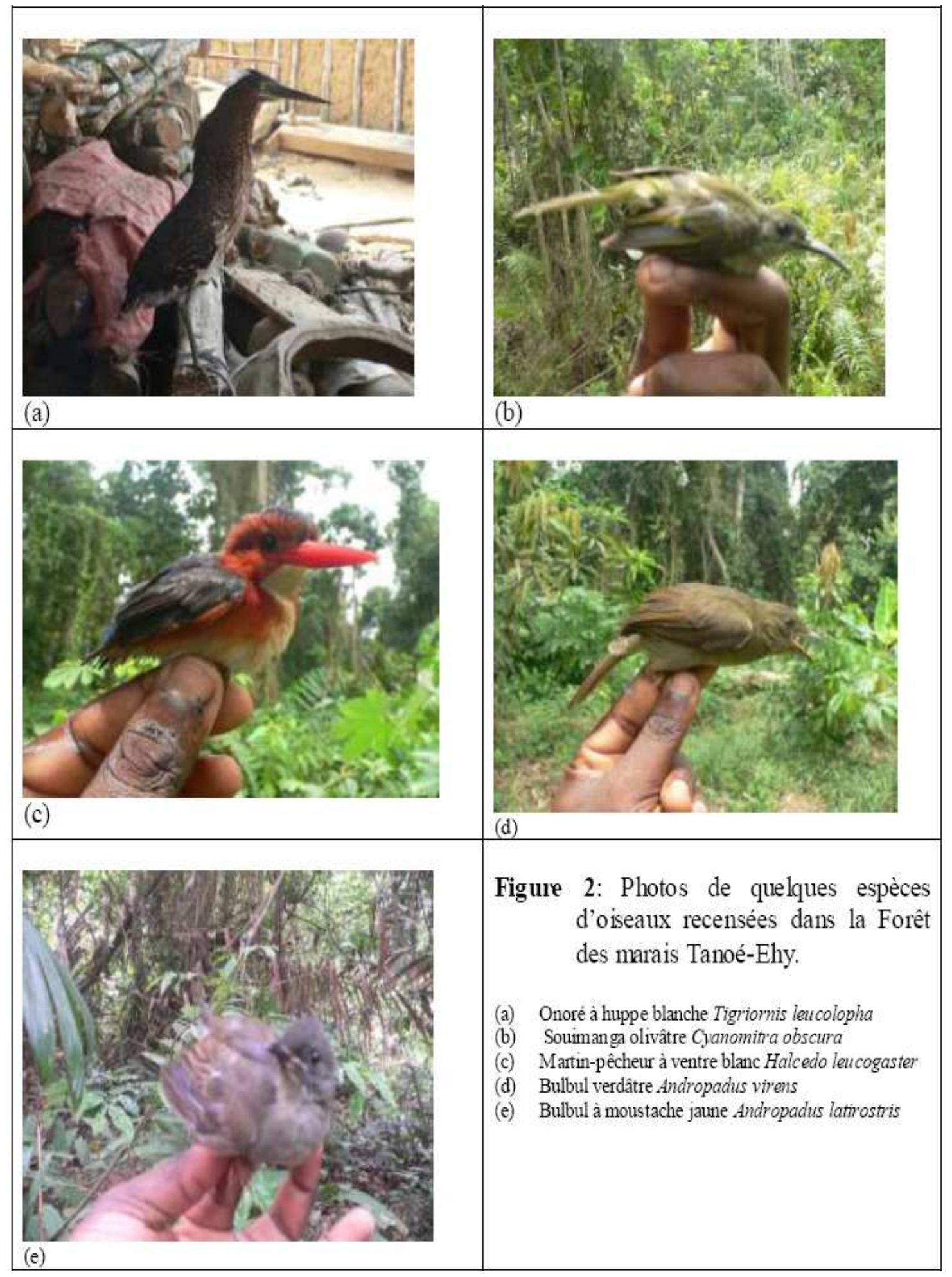


L'observation de la Chouette-pêcheuse de Bouvier Scotopelia bouvieri dans la FMTE, est très remarquable en ce sens qu'à notre connaissance, cette espèce semble être une nouvelle mention pour la Côte d'Ivoire. En effet, cette espèce dont l'aire de répartition connue couvre une bonne partie de l'Afrique centrale (Borrow et Demey, 2001) avait été observée au Nigéria, près de Lagos le long du fleuve Ogwé (Elgood, 1994) et celui d'Oni (Green et al., 2007), faisant de ce pays l'extrême ouest de son aire de répartition. L'observation de cette espèce dans la FMTE apparaît être l'un des résultats les plus plausibles de la présente étude.

La présence de la Chouette-pêcheuse rousse Scotopelia ussheri, espèce en danger, endémique en Afrique de l'Ouest (Ahon et Rondeau, 2008; Conway et al., 2010 ; BirdLife International, 2012), dont l'écologie et la biologie demeurent inconnues (Conway et al., 2010) et surtout rare en zone forestière côtière de Côte d'Ivoire (Ahon, 2010), témoigne davantage que la FMTE est en bon état de conservation. Cela indique que la FMTE représente un refuge pour la faune terrestre en général, et pour les oiseaux en particulier dans cette partie du territoire ivoirien. Aussi, le site dite «Plate-forme : 0519215 N / 0574988 O» de la FMTE, avec, une altitude assez élevée offrant un grand champ visuel d'observation de la FMTE, serait un endroit idéal pour la création d'un «Bird Watchsite » pour l'observation des mouvements migratoires des oiseaux en général et des rapaces en particulier, qui survolent cette région en saison favorable.

La présente étude a montré que la FMTE renferme une importante communauté d'oiseaux très diversifiée. En effet, n'ayant pas été explorée dans sa totalité, la FMTE pourrait sans doute abriter d'autres espèces non encore signalées en Côte d'Ivoire. La présence de cette importante communauté d'oiseaux pourrait s'expliquer par le caractère d'impraticabilité (zone marécageuse et fortement boueuse) de la FMTE. En effet, cette forêt est inondée presque tout au long de l'année et est difficilement accessible, même en saison sèche (Adou, 2007 ; Béné et al., 2012). Cependant, la FMTE fait l'objet de graves menaces (Béné et al., 2012), notamment le braconnage, l'exploitation forestière, les défrichements, etc.

\section{Conclusion}

$\mathrm{Au}$ terme de cette étude, il ressort que l'avifaune de la FMTE qui était méconnue avant la présente étude, est maintenant connue en partie. Ainsi, avec 274 espèces d'oiseaux, la FMTE renferme une importante communauté d'oiseaux très diversifiée avec des espèces typiquement forestières et migratrices, assez relativement importantes. Aussi, avec 12 espèces incluses dans la liste des espèces dont la protection est d'intérêt mondial, cinq espèces menacées, huit espèces à répartition restreinte, 15 espèces endémiques au bloc forestier de Haute Guinée de l'Afrique de l'Ouest et 110 espèces du biome des forêts guinéo-congolaises, la FMTE se qualifie comme ZICO selon les critères $\mathrm{A} 1, \mathrm{~A} 2$ et $\mathrm{A} 3$ définis par Fishpool et Evans (2001). Cependant, n'ayant pas été explorée dans sa totalité et les courtes périodes des inventaires, indiquent que la liste des espèces observées ne représente qu'une liste préliminaire de son avifaune. Des études complémentaires sont indispensables afin d'avoir une parfaite connaissance de l'avifaune de la FMTE.

\section{REMERCIEMENTS}

Nous tenons à remercier très sincèrement les responsables du RASAPCI et de SOS-Forêts qui ont mis à notre disposition les moyens nécessaires pour la réalisation de ce travail. Nous remercions également les populations riveraines des villages de Kongodjan, Yao-Akakro et Dohouan, et particulièrement nos guides IBRAHIM 
Mamadou MM, AKATIA Kassi (Rasta) et MONAHUE Bahota Jean, pour leurs appuis sans faille apportés dans le cadre de ce travail.

\section{REFERENCES}

Adou YCY. 2007. Mission d'inventaire préliminaire de la faune et de la flore de la Forêt des Marais Tanoé (13-27). Partie II : Inventaire préliminaire de la flore et description de la végétation. Rapport de mission CEPA/RASAP-CI, p. 34.

Ahon DB. 2010. Peuplement en rapaces de la zone forestière côtière de Côte d'Ivoire : distribution, statut de conservation et sensibilité de la Chouette-pêcheuse rousse Scotopelia ussheri (SHARPE, 1871) à la repasse de sa vocalisation. Thèse de Doctorat, Université de Cocody, Abidjan, Côte d'Ivoire, p.145.

Ahon DB, Rondeau G. 2008. Distribution, conservation et réponse à la provocation acoustique de la Chouette-pêcheuse rousse Scotopelia ussheri en zone forestière côtière de Côte d'Ivoire. Malimbus, 30: 143-144.

Béné J-CK, Koné I, Gonédélé Bi, Bitty EA, Ouattara K, Akpatou KB, N'guessan KA, Koffi DA. 2012. The diurnal primate community of the Tanoé Forest: species composition, relative abundance, distribution, polyspecific associations and conservation status. Int. J. Biol. Chem. Sci., 6(1): 51-64.

Bennun L, Howell K. 2004. Les oiseaux. In La Biodiversité des Forêts d'Afrique: Manuel Pratique de Recensement des Vertébrés, Bennun L, Davies G, Howell $\mathrm{K}$, Newing $\mathrm{H}$, Linkie M, Clarke J, Devitre D, Devitre R (eds). Earthwatch Institute: Royaume Uni; 135-180.

BirdLife International. 2010. Threatened Birds of the World. www.birdlife.net.

BirdLife International. 2012. Species factsheet: Scotopelia ussheri. www.birdlife.org (accessed April 2012).
Borrow N, Demey R. 2001. Birds of Western Africa. Christopher Helm: London.

Chappuis C. 2000. Les oiseaux de l'ouest africain. Sound supplement to Alauda. 15 CD-ROM. Société d'Etudes Ornithologiques, Paris.

Conservation International. 2001. De la forêt à la mer: les liens de la biodiversité de la Guinée au Togo. Priorités scientifiques régionales pour la conservation de la biodiversité. Conservation International, p. 78.

Conway A, Hernandez S, Carroll JP. 2010. Rufous Fishing Owl Scotopelia ussheri camera-trapped at midday near Tiwai Island, Sierra Leone. Bull. ABC, 17: 9091.

Demey R, Rainey H. 2005. Inventaire rapide des oiseaux des forêts classées de la Haute Dodo et du Cavally. In Une Evaluation Biologique de Deux Forêts Classées du Sud-Ouest de la Côte d'Ivoire, Alonso L, Lauginie F, Rondeau G (eds). Bulletin RAP d'Evaluation Rapide 34, Conservation International: Washington, D.C.; 76- 83.

Elgood JH. 1994. The Birds of Nigeria: an Annotated Check-list BOU Check-list No.4 ( $2^{\text {eme }}$ edn). Tring, British Ornithologists' Union.

Fishpool LDC. 2001. Côte d'Ivoire. In Important Bird Areas in Africa and Associated Islands: Priority Sites for Conservation, Fishpool LDC, Evans MI (eds). Pisces Publications: Newbury and BirdLife International: Cambridge, UK; 219-232.

Furness RW, Greenwood JJD. 1993. Birds as Monitors of Environmental Change. Chapman \& Hall: London, UK.

Green AA, Hall P, Leventis AP. 2007. Avifauna of the Omo Forest Reserve, SW Nigeria. Malimbus, 29: 16- 30.

Halle B, Bruzon V. 2006. Profil Environnemental de la Côte d'Ivoire. 
Rapport final, AGRIFOR Consult, Belgium, p. 128.

Icbp. 1992. Putting Biodiversity on the Map:

Priority Areas for Global Conservation.

International Council for Bird Preservation: Cambridge, UK.

Kouadio KP. 2006. Etude du peuplement avifaunique de la forêt classée de N'ganda-N'ganda à Assinie Mafia (sudest de la Côte d'Ivoire). DEA, Université de Cocody, Abidjan, Côte d'Ivoire, p. 2-3.

Lauginie F. 2007. Conservation de la nature et aires protégées en Côte d'Ivoire. NEI/Hachette et Afrique Nature, Abidjan, Côte d'Ivoire, p. 668.

Myers N, Mittermeier RA, Mittermeier CG, Fonseca GAB, Kent J. 2000. Biodiversity hotspots for conservation priorities. Nature, 403: 853-858.

Stattersfield AJ, Crosby MJ, Long AJ, Wege DC. 1998. Endemic Bird Areas of the World: Priorities for Biodiversity Conservation No 7. BirdLife International: Cambridge, UK.; p. 846.

Thiollay JM. 1985. The birds of Ivory Coast: status and distribution. Malimbus, 7: 159.

Thirgood SJ, Heath MF. 1994. Global patterns of endemism and the conservation of biodiversity. In Systematic and Conservation Evaluation, Forey PL, Humphries CJ, Vane-Wright RL (eds). Clarendon Press: Oxford, UK; 207-227. 